\title{
Isolation and Ultrastructural Characterization of Squid Synaptic Vesicles
}

\author{
GULCIN PEKKURNAZ ${ }^{1,2, *}$, ANDREA FERA ${ }^{2,3}$, JESSICA ZIMMERBERG-HELMS ${ }^{1,2}$, \\ JOSEPH A. DEGIORGIS ${ }^{2,3,4}$, LUDMILA BEZRUKOV ${ }^{1}$, PAUL S. BLANK ${ }^{1}$, JULIA MAZAR ${ }^{1,2}$, \\ THOMAS S. REESE ${ }^{2,3}$, AND JOSHUA ZIMMERBERG ${ }^{1,2, \dagger}$ \\ ${ }^{1}$ National Institute of Child Health and Human Development, NIH, Bethesda, Maryland 20892; \\ ${ }^{2}$ Marine Biological Laboratory, Woods Hole, Massachusetts 02543; ${ }^{3}$ National Institute of Neurological \\ Disorders and Stroke, NIH, Bethesda, Maryland 20892; and ${ }^{4}$ Biology Department, Providence College, \\ Providence, Rhode Island 02918
}

\begin{abstract}
Synaptic vesicles contain a variety of proteins and lipids that mediate fusion with the pre-synaptic membrane. Although the structures of many synaptic vesicle proteins are known, an overall picture of how they are organized at the vesicle surface is lacking. In this paper, we describe a better method for the isolation of squid synaptic vesicles and characterize the results. For highly pure and intact synaptic vesicles from squid optic lobe, glycerol density gradient centrifugation was the key step. Different electron microscopic methods show that vesicle membrane surfaces are largely covered with structures corresponding to surface proteins. Each vesicle contains several stalked globular structures that extend from the vesicle surface and are consistent with the V-ATPase. BLAST search of a library of squid expressed sequence tags identifies $10 \mathrm{~V}$ ATPase subunits, which are expressed in the squid stellate ganglia. Negative-stain tomography demonstrates directly that vesicles flatten during the drying step of negative staining, and furthermore shows details of individual vesicles and other proteins at the vesicle surface.
\end{abstract}

\section{Introduction}

Neurotransmitter release by fusion of synaptic vesicles with the pre-synaptic plasma membrane upon transient increases in intracellular $\mathrm{Ca}^{2+}$ is essential for propagating

Received 13 October 2010; accepted 3 March 2011.

* Present address: The F.M. Kirby Neurobiology Center, Children's Hospital Boston, and Department of Neurobiology, Harvard Medical School, MA 02115.

† To whom correspondence should be addressed. E-mail: joshz@ mail.nih.gov

Abbreviations: SV, synaptic vesicle. action potentials between neurons. Fusion of a synaptic vesicle (SV) with the pre-synaptic membrane requires cooperative interactions between the lipids and proteins of both membranes. Although the structure of many SV proteins has been solved and a prototypic structural model of an individual SV has been presented (Takamori et al., 2006), an overall picture of how proteins are organized at the vesicle surface is still lacking.

It is well established that the vertebrate and invertebrate nervous systems exhibit many similarities in neuronal function. The squid nervous system in particular has been used to demonstrate the neuronal resting potential as well as to record electrical action potentials. The squid was also used to define the role of calcium in synaptic transmission. The squid optic lobe contains $50 \%-80 \%$ of the neurons in the squid central nervous system and is therefore an excellent source of SVs for study of their biophysical and structural properties. Dowdall and Whittaker (1973) described the isolation of $\mathrm{SV}$-rich fractions from squid optic lobe by osmotic shock. However, the purity of their final fraction was never critically evaluated by either biochemical techniques or electron microscopy. Chin and Goldman (1992) used the same method to purify SVs from frozen squid optic lobe and added controlled-pore glass chromatography as a final purification step. Their detailed biochemical analysis indicated that the vesicle fraction was about $60 \%$ pure.

Using advances in the purification of SVs from rat brain (Huttner et al., 1983), we optimized an SV isolation protocol for squid (Logilo pealei [LeSueur, 1821]) optic lobe in this study to obtain a highly pure and intact SV population for biochemical and ultrastructural studies. The SV-enriched fractions were analyzed by electron microscopy 
(EM) to evaluate their purity and size distribution as well as the effects of different specimen preparation techniques on the average SV size. The distribution of SV size in SVenriched fractions suggests that the SV we isolated are more than $95 \%$ pure. Finally, the purified vesicles were used to characterize the organization of the surface of individual SVs by tungstate-based negative-stain EM tomography. Here we present a three-dimensional molecular rendering of the surface structure, presumably V-ATPase, reflecting an individual SV, rather than the average of many SVs.

\section{Materials and Methods}

\section{Preparation of synaptosomes}

Live squid (Loligo pealei) obtained from the Marine Resources Center, Marine Biological Laboratory, Woods Hole, Massachusetts, were maintained for 2 to $24 \mathrm{~h}$ in tanks containing running seawater at $16-22{ }^{\circ} \mathrm{C}$. All further manipulations were in ice-cold buffers containing $0.01 \%$ 2,6Di-tert-butyl-4-methylphenol (BHT) to prevent lipid oxidation. Squid optic lobes were dissected onto pre-chilled petri dishes and weighed. Synaptosomal fractions were prepared as previously described (Dowdall and Whittaker, 1973; Pollard et al., 1975), with modification to produce an isotonic homogenization buffer $\left(1 \mathrm{~mol} \mathrm{l}^{-1}\right.$ sucrose, $5 \mathrm{mmol}^{-1}$ HEPES, 10 mmol $\mathrm{l}^{-1}$ EDTA/EGTA pH 7.4 with protease inhibitor cocktail [Roche Applied Science, Indianapolis, IN]). Squid optic lobes were homogenized as a $20 \%(w / v)$ solution in $1 \mathrm{~mol} \mathrm{l}^{-1}$ sucrose buffer by $6-8$ complete strokes of a glass-on-glass TenBroek homogenizer with pestle B. The homogenate $(\mathrm{H})$ was centrifuged at $13,000 \times g$ for $1 \mathrm{~h}$ at $4{ }^{\circ} \mathrm{C}$ in a JA-20 rotor. The latter step yielded a pellet (P1; mitochondrial fraction), a floating particulate layer (PL; synaptosomal fraction), and an opaque supernatant (S1; microsomal fraction, see Fig. 1A). The synaptosomal fraction was collected and stored on ice for further fractionation.

\section{Purification of synaptic vesicles}

The synaptosomal fraction was diluted in ice-cold distilled water supplemented with protease inhibitor cocktail (Roche Applied Science, Indianapolis, IN) (2 ml/g of synaptosomal tissue), and then resuspended with a glass Pasteur pipette. The synaptosomal lysate (L) was adjusted with 10 mmol $1^{-1}$ HEPES (pH 7.3) and 10 mmol $1^{-1}$ EDTA and incubated for $20 \mathrm{~min}$, followed by centrifugation at $21,000 \times g$ for $20 \mathrm{~min}$. The supernatant (LS1) was collected and centrifuged at 195,000 $\times g$ for $2 \mathrm{~h}$.

The pellet (LP2) containing the crude SV fraction was resuspended in $0.4 \mathrm{~mol} \mathrm{l}^{-1}$ sucrose and homogenized by passing through a 25 -gauge needle (10 times). The resuspended vesicles were layered on to a sucrose step gradient $\left(0.4,0.6,0.8,1\right.$, and $1.2 \mathrm{~mol}^{-1}$ sucrose) and centrifuged for $2 \mathrm{~h}$ at 54,000 $\times g$ using a Beckman SW41Ti swing-bucket rotor. Purified SVs concentrated in the $0.4 \mathrm{~mol} \mathrm{l}^{-1}$ sucrose band (SG-V), were aspirated, diluted into squid $1 / 2 \times$ buffer (175 $\mathrm{mmol} \mathrm{l}^{-1}$ aspartic acid, $65 \mathrm{mmol} \mathrm{l}^{-1}$ taurine, $85 \mathrm{mmol}$ $\mathrm{l}^{-1}$ betaine, $25 \mathrm{mmol} \mathrm{l}^{-1}$ glycine, $10 \mathrm{mmol} \mathrm{l}{ }^{-1}$ HEPES, 6.5 mmol $\mathrm{l}^{-1} \mathrm{Mg}_{2} \mathrm{Cl}, 5 \mathrm{mmol} \mathrm{l}^{-1}$ EGTA pH 7.2) and centrifuged at $198,000 \times g$ for $1 \mathrm{~h}$ to obtain a vesicle pellet. The synaptic vesicle pellet (SVP) resuspended with squid $1 / 2 \times$ buffer was homogenized by passing through a 25 -gauge needle and then loaded onto a continuous glycerol gradient (Clift-O'Grady et al., 1990).

\section{Immunoblots}

Bicinchoninic acid (BCA) assay kits (Thermo Scientific, Pierce Biotechnology, Rockford, IL) were used to measure total protein concentration in fractions, using bovine serum albumin (BSA) as a standard. Samples were boiled in gel loading buffer containing 5\% 2-mercaptoethanol (Sigma Chemical Co., St. Louis, MO), and immediately transferred to ice. SDS-PAGE was carried out using 4\%-20\% trisglycine gel (NOVEX, San Diego, CA), and $5 \mu \mathrm{g}$ of total protein/sample. Proteins were transferred to PVDF (polyvinylidene fluoride) membranes $(0.2-\mu \mathrm{m}$ pore size). Blots were blocked in phosphate-buffered saline (PBS), $\mathrm{pH}$ 7.2, containing 5\% nonfat milk and $0.05 \%$ Tween-20 for $1 \mathrm{~h}$ with gentle agitation. Anti-SNAP-25 (Alomone Labs Ltd., Jerusalem, Israel) and anti-VDAC (N18; Santa Cruz Biotechnology, Inc., Santa Cruz, CA) antibodies were diluted in block (1:1000), and blots were probed for $60 \mathrm{~min}$. Blots were rinsed 4 times for $15 \mathrm{~min}$ each in PBS-0.05\% Tween-20 (PBST) and then incubated in alkaline phosphatase-conjugated secondary antibodies diluted in block (1:8000) for $1 \mathrm{~h}$. Blots were rinsed in PBST 4 times for 15 min with agitation and developed with ECF substrate (GE Healthcare UK Limited, Little Chalfont, Buckinghamshire, UK). Blots were visualized using a Fujifilm FLA3000 scanner, and band intensities quantified using Image Gauge software, ver. 3.46, Fuji Photo Film Co. LTD.

\section{Sample preparation for electron microscopy}

Chemical fixation: Synaptosomal fractions were centrifuged at $10,000 \times g$ for $5 \mathrm{~min}$ in $1 \%$ BSA to form a sticky pellet. Pellets were fixed in $3 \%$ glutaraldehyde, $0.5 \%$ acrolein $(1: 2$; synaptosomal pellet/fixative) and post-fixed in $1 \%$ osmium in water. Fixed pellets were washed with $0.1 \mathrm{~mol}^{-1}$ sodium acetate buffer ( 3 times), and $1 \mathrm{~mol} \mathrm{l}^{-1}$ sucrose (1 time), dehydrated through a series of ethanol solutions, embedded in araldite, sectioned, and stained conventionally.

Negative stain: Drops $(10 \mu \mathrm{l})$ of each SV fraction were placed on Parafilm in a covered, humidified petri dish. Freshly glow-discharged, Formvar-coated, 400-mesh copper grids (SPI Supplies) were incubated for $10 \mathrm{~min}$ on the SV drops, washed three times for 10 min each in squid $1 / 2 \times$ buffer, and stained with $1 \%$ uranyl acetate in water. 
A

\begin{tabular}{|c|c|}
\hline HOMOGENIZATION & $\begin{array}{l}20 \%(\mathrm{w} / \mathrm{v}) \text { squid optic lobe tissue } \\
\text { in buffered } 1 \mathrm{M} \text { sucrose } \\
\text { Homogenate }(\mathbf{H}) \\
\begin{array}{l}13000 \mathrm{~g} 1 \mathrm{~h} 4^{\circ} \mathrm{C} \\
\end{array}\end{array}$ \\
\hline $\begin{array}{l}\text { DIFFERENTIAL } \\
\text { CENTRIFUGATION }\end{array}$ & $\begin{array}{l}\text { Floating pellicle, } \\
\text { synaptosomes (PL) } \\
\text { Supernatant (S1) } \\
\text { Pellet (P1) }\end{array}$ \\
\hline $\begin{array}{l}\text { LYSIS OF } \\
\text { SYNAPTOSOMES }\end{array}$ & $\begin{array}{l}\text { Resuspension of } \mathrm{PL} \text { with } \mathrm{ddH}_{2} \mathrm{O} \\
(2 \mathrm{ml} / \mathrm{g}) \text {, lysis } 20 \mathrm{~min} \text { at } 4^{\circ} \mathrm{C} \\
\text { Lysate (L) }\end{array}$ \\
\hline $\begin{array}{l}\text { DIFFERENTIAL } \\
\text { CENTRIFUGATION }\end{array}$ & $\begin{array}{l}\qquad 21000 \mathrm{~g} 20 \mathrm{~min} 4^{\circ} \mathrm{C} \\
\text { Lysis pellet (LP1) } \\
\text { Lysis supernatant (LS1) } \\
\text { Lysis supernatant (LS2) } \\
\text { Crude synaptic vesicle pellet (LP2) }\end{array}$ \\
\hline HOMOGENIZATION & $\begin{array}{l}\text { Resuspension of LP2 with } 0.4 \mathrm{M} \\
\text { sucrose and homogenization by } \\
\text { passing through } 25 \text { ' gauge needle }\end{array}$ \\
\hline $\begin{array}{l}\text { DISCONTINUOUS } \\
\text { SUCROSE } \\
\text { GRADIENT } \\
\text { DENSITY } \\
\text { GRADIENT } \\
\text { CENTRIFUGATION }\end{array}$ & $\begin{cases}54000 \mathrm{~g} 2 \mathrm{~h} 4^{\circ} \mathrm{C} \\
0.4 \mathrm{M} \\
0.6 \mathrm{M} \longrightarrow \\
0.8 \mathrm{M} & \begin{array}{l}\text { Synaptic vesicle } \\
\text { rich fraction } \\
1 \mathrm{M} \\
\text { (SG-V) } \\
1.2 \mathrm{M}\end{array} \\
\end{cases}$ \\
\hline $\begin{array}{l}\text { DIFFERENTIAL } \\
\text { CENTRIFUGATION }\end{array}$ & $\begin{array}{l}\downarrow_{\text {SG-V supernatant }} 198000 \mathrm{~g} 1 \mathrm{~h} 4^{\circ} \mathrm{C} \\
\text { SG-V pellet (SVP) }\end{array}$ \\
\hline HOMOGENIZATION & $\begin{array}{l}\text { Resuspension of SVP with } 1 / 2 X \text { buffer } \\
\text { and homogenization by passing through } \\
25 \text { ' gauge needle }\end{array}$ \\
\hline $\begin{array}{l}\text { CONTINUOUS } \\
\text { GLYCEROL } \\
\text { GRADIENT } \\
\text { DENSITY } \\
\text { GRADIENT } \\
\text { CENTRIFUGATION }\end{array}$ & $\downarrow \underbrace{218000 \mathrm{~g} 75 \mathrm{~min} 4^{\circ} \mathrm{C}}_{0.5 \mathrm{ml} 50 \% \text { sucrose }} \begin{array}{l}5-25 \% \text { glycerol } \\
\text { gradient }\end{array}$ \\
\hline
\end{tabular}

$\mathrm{B}$

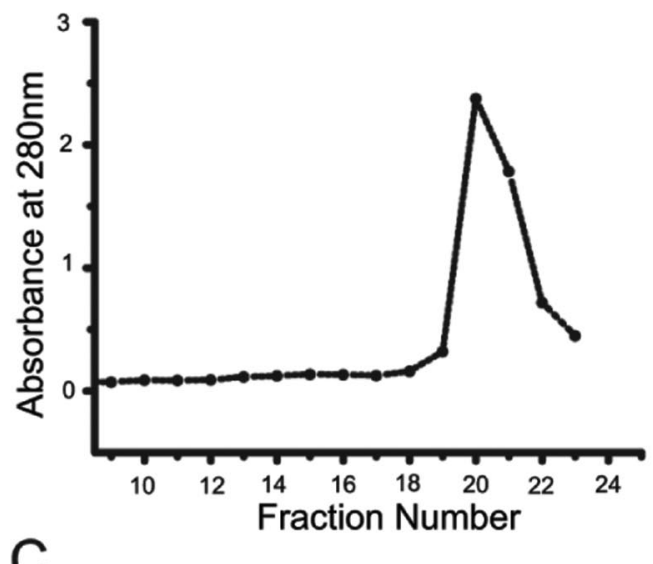

C

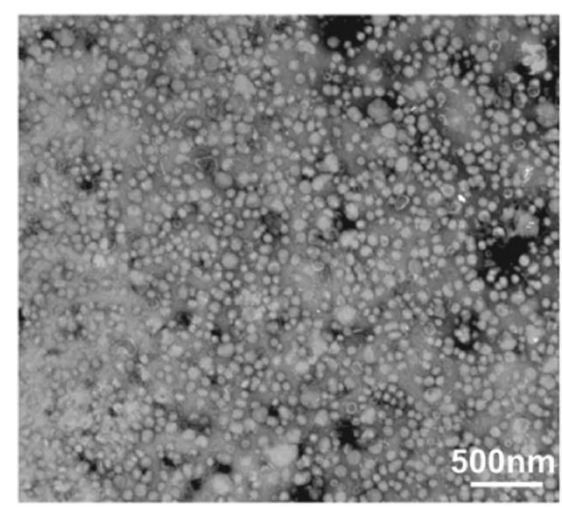

$\mathrm{D}$

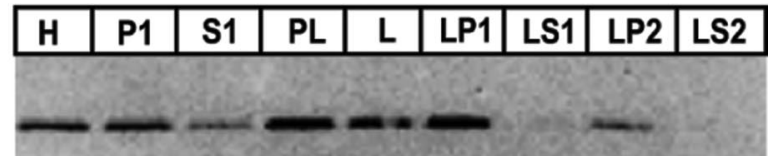

Anti-SNAP25
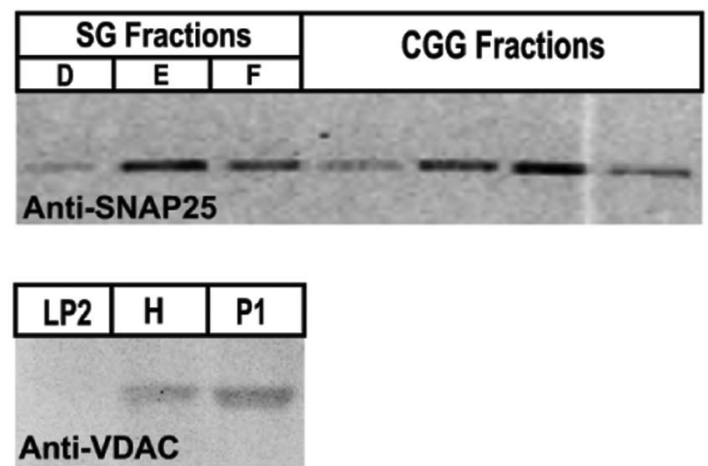

Figure 1. Synaptic vesicle enrichment by glycerol velocity sedimentation. (A) Scheme summarizing purification of synaptic vesicles from squid optic lobes. (B) Glycerol gradient fractions $(250 \mathrm{ml})$ collected from top to bottom and analyzed for their absorbance at $280 \mathrm{~nm}$. Major peak indicates the synaptic vesicle-rich fraction. (C) Electron micrograph of the synaptic vesicle-rich fraction. (D) Western blot of subcellular fractions obtained during the synaptic vesicle purification steps (as described in Materials and Methods) with mitochondrial marker Anti-VDAC (40 $\mu \mathrm{g}$ protein/lane) and synaptic marker Anti-SNAP25 (5 $\mu \mathrm{g}$ protein/lane) antibodies. 
Cryofixation: The synaptosomal fraction and SV fractions were centrifuged respectively at $13,000 \times g$ for $5 \mathrm{~min}$ and $198,000 \times g$ for $1 \mathrm{~h}$ in $1 \%$ BSA. Samples were rapid-frozen with a Life Cell CF-100 freeze-slam apparatus (Life Cell; The Woodlands, TX), freeze-substituted (Petersen et al., 2003), embedded, and thin-sectioned. Sections were stained routinely with uranyl acetate and lead citrate.

\section{Tomography}

Freshly glow-discharged copper grids coated with Formvar and carbon were floated on drops of SV preparation. Fiduciary markers were then added by floating the grids for 10 min on a $10-\mu 1$ drop of goat anti-rabbit antibody linked to $10 \mathrm{~nm}$ gold particles (Ted Pella, Inc., Redding, CA). Finally, grids were negative stained by floating them for 1 min on a $10-\mu l$ drop of $2.0 \%$ methylamine tungstate (Nano-W, Nanoprobes, Inc., Yaphank, NY). Tomography series were collected at $300 \mathrm{KV}$ in a Tecnai TF30 transmission electron microscope equipped with a $2048 \times 2048$ pixel Ultrascan, charge-coupled device (CCD) camera from Gatan at a pixel size of $0.29 \mathrm{~nm}$. Images were collected at each $2^{\circ}$ of tilt $\pm 60^{\circ}$ in orthogonal $X$ and $Y$ axes. NanoW is unchanged by the radiation from this tomography protocol (A. Fera, unpubl.).

Images from each axis were aligned separately to subpixel accuracy using IMOD, ver. 4.1.8, with the 10-nm gold particles serving as fiduciary markers (Kremer et al., 1996). The two tilt series were then combined into a tomogram at an accuracy of 0.3 pixels, the upper limit for the acceptable residual warping error in IMOD. Virtual sections, one voxel thick, were derived from the tomograms and then binned by two for further examination. ImageJ, ver. 1.44n, the public domain NIH Image program (http://rsb.info.nih.gov/nihimage/), was used to display virtual sections for evaluation and measurement. Data were spatially filtered and modified only for brightness and contrast.

\section{Morphometric analysis of synaptic vesicles}

Images were collected with a CCD digital camera system (XR-100 from AMT, Danvers, MA) at a microscope magnification of $40,000 \times$ and examined using ImageJ.

\section{Identification of V-ATPase transcripts in squid neuronal tissues}

Previously, we generated a database of squid expressed sequence tags (ESTs) by single-pass sequencing 22,689 cDNA clones derived from mRNA of the stellate ganglia of L. pealei (DeGiorgis et al., unpubl. data). These ESTs were assembled into contigs and singletons to yield 10,027 unique sequences, and each sequence was analyzed by BLASTX. The resulting analysis was scanned for subunit transcripts that are known to contribute to the functional V-ATPase (see Table 2).

\section{Results}

\section{Characterization of the synaptic vesicle-enriched fraction}

The purification procedure using homogenization of tissue, differential centrifugation, sucrose gradient flotation, and chromatography on a controlled-pore glass column provided an SV-enriched fraction of $60 \%$ purity (Chin and Goldman, 1992). To obtain intact vesicles with higher purity, freshly dissected optic lobe tissue was further fractionated by continuous glycerol density gradient centrifugation (Fig 1A). The density of closed vesicles on density gradients of membrane-impermeable solutes, such as sucrose, differs from the density measured using the density gradient of membrane-permeable substances such as glycerol (Carlson et al., 1978). A vesicle peak obtained after the glycerol gradient was enriched in a homogeneous population of intact vesicles (Fig. 1B and C), within which membrane pieces and large microsomes were very rare.

A SNAP-25 antibody was used to probe Western blots of fractions obtained during the purification steps, revealing a single band of immunoreactivity at $\approx 30 \mathrm{kDa}$ (Fig. 1D). Comparison of subcellular fractions indicated that SNAP25 was enriched in the synaptosomal fraction (PL) compared to the initial homogenate (H). SNAP-25 was present in the SV fractions but not enriched relative to the other fractions, in conformity with observations on rat brain (Takamori et al., 2006). However, the vesicle peak obtained following the glycerol gradient showed SNAP-25 enrichment relative to the other fractions. SV-rich fractions of rat brain contain the SV integral membrane proteins Synaptotagmin and SV2 as well as SNAP-25 (Kretzschmar et al., 1996). Other SV proteins could not be analyzed due to the lack of suitable antibodies. VDAC protein was used as a mitochondrial marker to demonstrate that there was no detectable mitochondrial contamination even in the crude SV fraction (LP2) (Fig 1D). As expected, the recovery of SV-enriched fraction is low $(0.03 \pm 0.01, n=4)$ due to the osmotic shock treatment and further fractionation with glycerol, a membrane-permeable solute used to eliminate broken vesicles (Table 1). Yields were $\approx 0.3 \mathrm{mg}$ of SV from $11 \mathrm{~g}$ of optic lobe tissue from 40-50 squid. We further evaluated the purity of this fraction based on SV diameter measurements.

The outer diameter has traditionally been used as a metric for SV size. Here, we measured the diameter of SV by outlining the outer SV membrane and then calculating the longest distance between any two points along the selection boundary (Feret's diameter). In addition, we determined circularity by using the built-in routines found in ImageJ software. Each vesicle counted had a clear membrane border and distinct negative staining. The calculated circularity value was greater than $0.998(n=5000)$, indicating that the SVs were uniform in their horizontal and vertical dimensions. We further evaluated the probability distribution of measured diameters to assess the level of contamination. A 
Table 1

Distribution and percent recovery of protein in subcellular fractions obtained in the course of purification of synaptic vesicles from squid optic lobe (11 g optic lobe tissue, mean $\pm S D, n=4)$

\begin{tabular}{lcc}
\hline \hline & \multicolumn{2}{c}{ Total protein } \\
\cline { 2 - 3 } Subcellular fraction & Amount $(\mathrm{mg})$ & Recovery $(\%)$ \\
\hline H & $1041.45 \pm 184.68$ & 100.00 \\
PL & $144.28 \pm 84.22$ & $16.91 \pm 1.75$ \\
S1 & $318.04 \pm 50.94$ & $30.61 \pm 1.02$ \\
P1 & $354.14 \pm 73.06$ & $34.70 \pm 9.26$ \\
L & $201.01 \pm 55.25$ & $18.72 \pm 3.43$ \\
LP1 & $100.08 \pm 23.91$ & $10.03 \pm 3.46$ \\
LS1 & $44.46 \pm 10.31$ & $4.39 \pm 1.4$ \\
LP2 & $8.91 \pm 2.22$ & $0.89 \pm 0.33$ \\
LS2 & $32.55 \pm 5.41$ & $3.19 \pm 0.67$ \\
SG-E & $2.78 \pm 3.54$ & $0.27 \pm 0.38$ \\
SG-F & $2.10 \pm 0.72$ & $0.21 \pm 0.1$ \\
SG-E supernatant & $1.13 \pm 1.56$ & $0.12 \pm 0.17$ \\
CGG-19 & $0.17 \pm 0.02$ & $0.02 \pm 0$ \\
CGG-20 & $0.32 \pm 0.13$ & $0.03 \pm 0.01$ \\
CGG-21 & $0.25 \pm 0.2$ & $0.02 \pm 0.02$ \\
\hline
\end{tabular}

single Gaussian fit of the frequency histogram was used to calculate mean SV diameter and standard deviation. Values larger than the mean plus four standard deviations $(99.994 \%$ confidence interval) were taken as an indication of contamination of the $\mathrm{SV}$ fraction, and results show that the purity of the SV-enriched fraction was $96 \pm 2.6 \%$ (mean $\pm \mathrm{SD}$, $n=20$ different isolations). The same analysis was performed for area and volume values. There was no significant difference between the three types of analysis; therefore we used the diameters as a measure for SV size.

\section{Effect of specimen preparation method on synaptic vesicle diameter}

Accurate assessment of SV size within pre-synaptic nerve terminals and in SV-enriched fractions requires sample preparation for electron microscopy and morphometric analysis. The size distribution of SVs at intact nerve endings (synaptosomes) and within SV-enriched fractions was compared using different preparation techniques to determine the mean diameter of SVs precisely (Fig. 2). Our morphometric analysis results revealed that the vesicle size distribution was significantly different according to sample preparation technique (ANOVA, $P<0.001$, Fig. 2). The outer diameter of SVs in synaptosomes, excluding dense-core vesicles, was compared. In fixed and rapid-frozen synaptosome samples, mean diameters were $47.8 \pm 0.7 \mathrm{~nm}$ (mean $\pm \mathrm{SEM}, n=142)$ and $52.1 \pm 0.8 \mathrm{~nm}($ mean $\pm \mathrm{SEM}$,
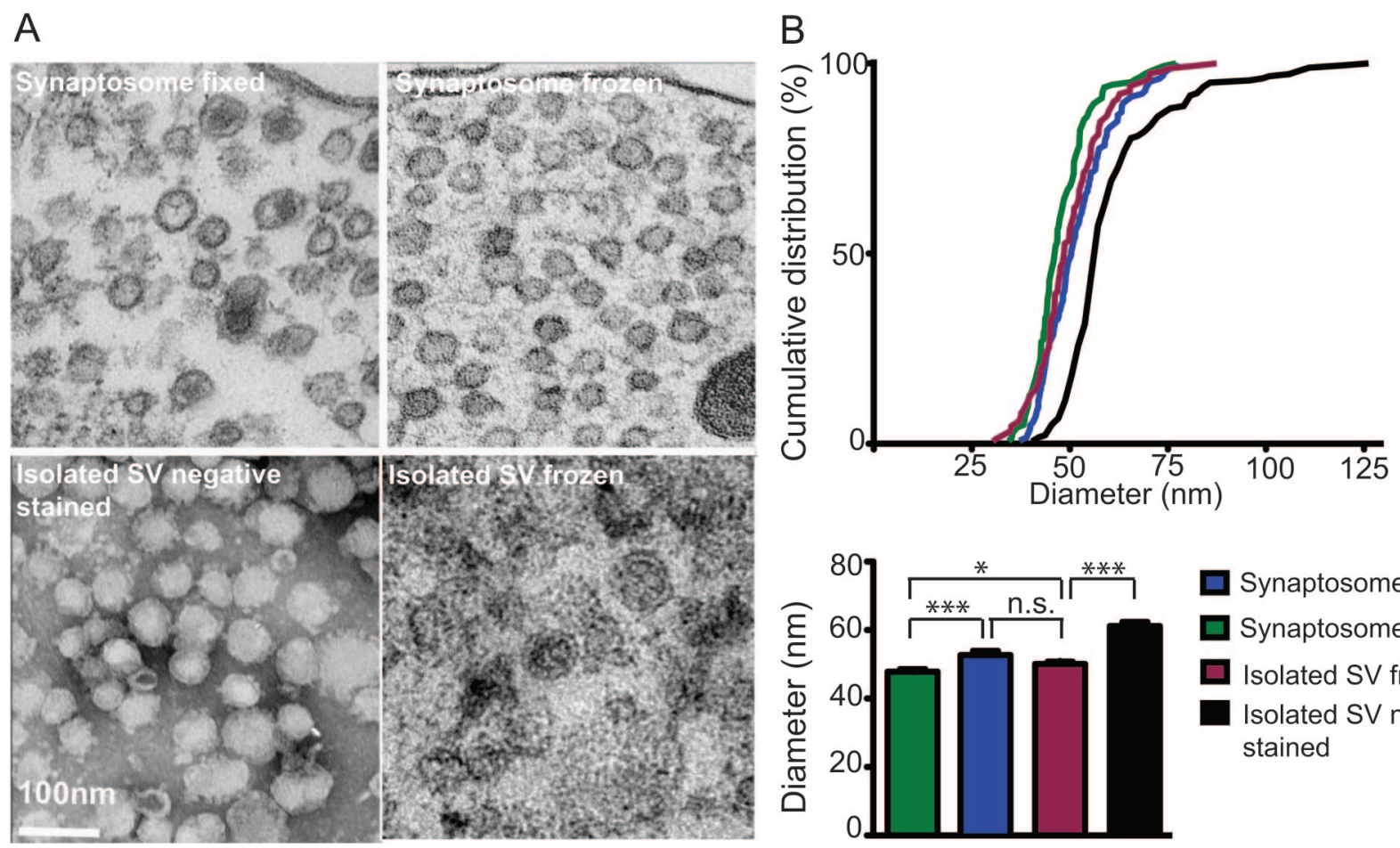

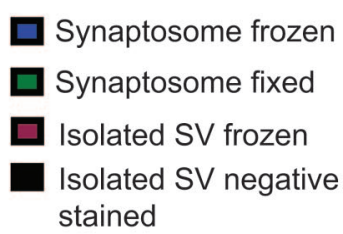
stained

Figure 2. Comparison of squid synaptic vesicle (SV) size by altering electron microscopy sample preparation technique in synaptosomes and isolated vesicles. (A) Electron micrograph of (left to right) fixed, embedded, thin-sectioned synaptosome; slam-frozen, freeze-substituted synaptosome; negative stained isolated SVs; and slam-frozen, freeze-substituted isolated SVs. (B) Cumulative distribution of SV diameters. Variations in mean diameter due to different sample preparation regimens (mean \pm SEM, $n=125-175$, Student's $t$-test; “*” $=P<0.03$, “****" $=P<0.001$, n.s. $=$ not significant $)$. 
$n=125$ ), respectively. Shrinkage during aldehyde fixation as well as rounding and swelling of vesicles due to subtle ice crystal formation during rapid freezing could explain a small difference in SV diameter (Student's $t$-test, $P<$ 0.001). A visible pellet of the SV-enriched fraction was used to prepare a rapid-frozen sample, which revealed an $\mathrm{SV}$ diameter of $50.1 \pm 0.8 \mathrm{~nm}$ (mean \pm SEM, $n=125$ ), very similar to the value obtained from SVs in slam-frozen synaptosome samples.

For further evaluation, the size distributions of freshly isolated, negatively stained (with uranyl acetate) SVs were compared to those of other samples. Negative staining theoretically replaces water in the interstices of the object until all hydrated volumes are filled with stain, thus forming a uniformly thin, amorphous film in which the specimen is supported and preserved. Negatively stained samples had the largest mean diameter, $61.2 \pm 1.1 \mathrm{~nm}$ (mean \pm SEM, $n=178$ ), compared to the other samples (Student's $t$-test, $P<0.001$ ) (Fig. 2). The larger diameter can be explained by flattening of the vesicles upon drying down in the negative stain (see Discussion).

\section{Molecular structure of synaptic vesicles}

Knob-like protrusions found on the surfaces of SVs, apparent after negative staining, have previously been regarded as the vacuolar ATP-dependent proton pump (VATPase) (Wilkens et al., 2005; Takamori et al., 2006). V-ATPases are large, membrane-bound multi-subunit complexes that translocate protons across membranes. The number of V-ATPase structures per vesicle was variable, with most vesicles containing four or five (Fig. 3). Correlation analysis failed to detect a linear relationship between vesicle size and V-ATPase number (data not shown). Guinea pig (Stadler and Tsukita, 1984) and rat (Takamori et al., 2006) SVs contain one or two and occasionally three or four V-ATPases per vesicle. Although the density of V-ATP structures in squid SV is greater than reported values, the size of the individual units, $15-20 \mathrm{~nm}$ in diameter, is in good agreement.

Electron microscopy of synaptic vesicles negatively stained with Nano-W served to further evaluate molecular structures at SV surfaces. The negative stain methylamine tungstate tolerates collection of the 140 images typically needed for a tomography series, yielding reconstructions of SV surfaces with exquisite detail. Seven tomograms, corresponding to 10 vesicles, were examined and analyzed in virtual sections calculated from tomograms. Overlap of images of individual proteins on the surfaces of the SVs is eliminated by the calculation of virtual sections along three arbitrary perpendicular axes (Kremer et al., 1996). Surfaces of vesicles, as expected, displayed numerous structures presumed to be proteins. There are no indications from measurements of diameters that stain penetrates inside SVs, suggesting that the mixture of proteins and lipids on the

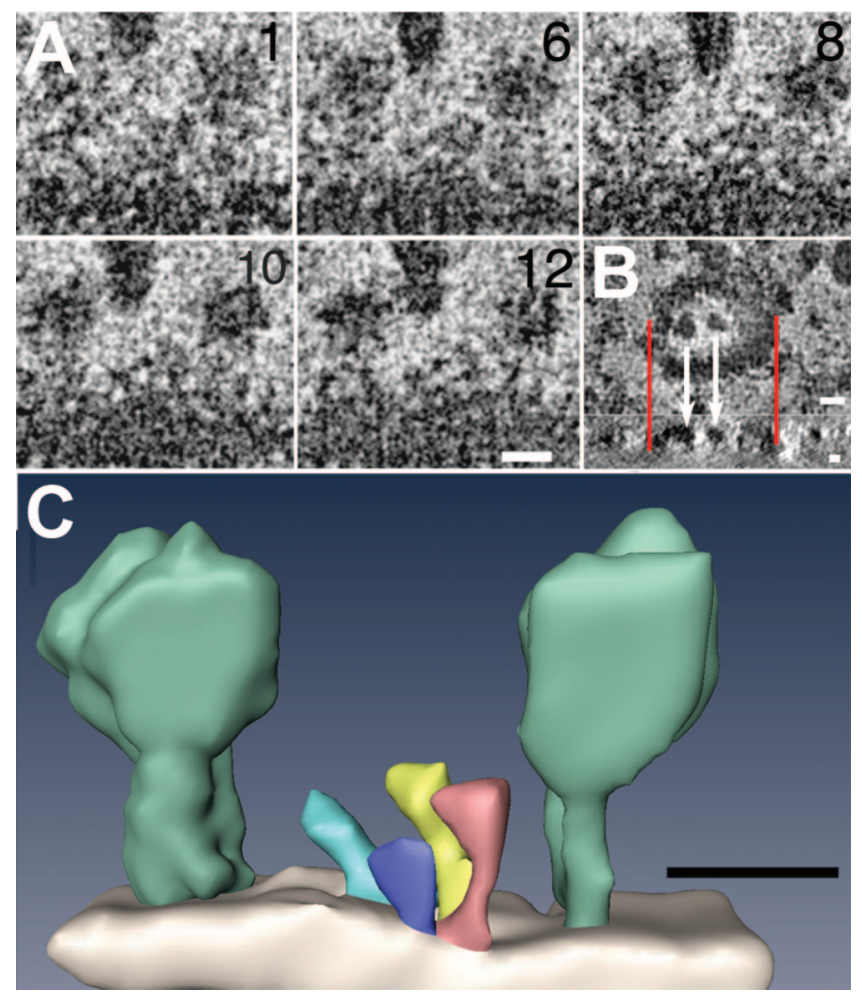

Figure 3. (A) Five virtual sections (1, 6, 8, 10, and 12), each 0.6-nm thick, extracted from different levels in a tomogram of a single negativestained synaptic vesicle. The external surface of the vesicle displays three prominent molecules. Data have been adjusted for brightness and contrast, binned by two, and spatially filtered to increase the signal-to-noise. (B) Virtual section through vesicle in en-face plane showing how two large surface particles (white arrows) aligned with a cross sectional projection below. Synaptic vesicles collapse during negative staining, leaving other prominent molecules on their collapsed external surfaces exposed to negative stain. Red lines delineate two edges of vesicle above and the same edges below. Surface particles lie in a plane defined by the two edges of the vesicle, showing that the vesicle is flattened onto the substrate (below). $($ Scale bar $=10 \mathrm{~nm})$. (C) Corresponding surface rendering of structures on the surface of the vesicle in A, including two of the more prominent ones (light green) whose sizes and shapes correspond to EM reconstructions of the V-ATPase of Thermus thermophilus (Bernal and Stock, 2004). Smaller structures (other colors) are also evident on the surface of the vesicle. $($ Scale bar $=10 \mathrm{~nm})$.

surface of SVs is not affected by the processing for electron microscopy. Therefore, the protein coat appears to be distributed uniformly on the surface of the SV rather than in tight clusters within restricted domains as reported previously (Bennett et al., 1992), since we do not see open membrane patches. One exception to this appearance of even distribution is the few much larger surface components displaying a size and shape consistent with the V-ATPase (Fig. 3).

Squid V-ATPase showed structural similarity to its mammalian homologs (Wilkens et al., 2005; Takamori et al., 2006). There are very few sequences corresponding to squid proteins in public databases, but squid data (DeGiorgis et al., unpubl. data) containing 23,000 ESTs from Loligo pea- 
Table 2

Cross species sequence comparisons of V-ATPase transcripts identified in squid neuronal tissues*

\begin{tabular}{llcc}
\multicolumn{1}{c}{ Top BLASTx } & Accession No. Species & Match location & Identity/Similarity $(\%)$ \\
\hline \hline $\mathrm{V}_{1}$ Catalytic Subunit a & Q7sy46 zebrafish & $13-266$ & $80 / 90$ \\
$\mathrm{~V}_{1}$ Catalytic Subunit a & Q90647 chick & $404-617$ & $81 / 92$ \\
$\mathrm{~V}_{1}$ Catalytic Subunit c & Q291s7 fruitfly & $1-29$ & $61 / 76$ \\
$\mathrm{~V}_{1}$ Subunit d & Q6azj2 frog & $5-192$ & $79 / 91$ \\
$\mathrm{~V}_{1}$ Subunit e & Qhqt6 mosquito & $1-224$ & $67 / 82$ \\
$\mathrm{~V}_{1}$ Subunit g & Q59gy4 oyster & $7-60$ & $55 / 57$ \\
$\mathrm{~V}_{1}$ Subunit $\mathrm{h}$ & Q7qg40 mosquito & $228-447$ & $67 / 80$ \\
$\mathrm{~V}_{1}$ Subunit h & Q7qg40 mosquito & $4-210$ & $56 / 76$ \\
$\mathrm{~V}_{0}$ 16 kd proteolipid subunit c & Q6p041 zebrafish & $1-153$ & $77 / 83$ \\
$\mathrm{~V}_{0}$ 21 kd proteolipid subunit c & Q6pd81 zebrafish & $2-203$ & $67 / 79$ \\
\hline
\end{tabular}

* Data from DeGiorgis et al. (unpubl.).

lei were used to search for nucleotide sequences corresponding to the V-ATPase peptides. The tBLASTn algorithm running locally pinpointed 10 ESTs that showed significant, but not exact, alignment cross species (Table 2). Conserved domains in V-ATPase subunits are thus in agreement with the observed structural homology.

\section{Discussion}

Dowdall and Whittaker (1973) described the isolation of a synaptic vesicle-rich fraction from squid optic lobe. However, the purity of the SV-rich fraction was not evaluated by either biochemical or electron microscopic techniques. Subsequently, SVs purified by this method from frozen squid optic lobe with the additional purification step of controlledpore glass chromatography yielded a purity of about $60 \%$ (Chin and Goldman, 1992). Since rat brain SV can be purified to greater than 95\% (Huttner et al., 1983), we modified the purification scheme for rat brain to isolate highly pure SVs from fresh squid optic lobes. Determinations of SV size distributions in SV-enriched fractions suggest that this isolation protocol provides SVs with a purity higher than $95 \%$ from squid optic lobes.

The results presented here clearly demonstrate that the estimates of SV size are dependent upon the method of preparation of the SV sample for electron microscopy. Section thickness is unlikely to be a source of variation because we only measured vesicles for which the delimiting edges of the membrane were visible in a single section and then computed the size based on Feret's diameter with a circularity value close to unity. Fixation and processing conditions can alter the absolute dimensions of organelles (Fox, 1988; Tatsuoka and Reese, 1989). In mammalian cholinergic synapses, the rapid-frozen, freeze-substituted SV diameter is also significantly greater than that obtained by aldehyde fixation, presumably because the osmotic effects of fixatives are avoided (Tatsuoka and Reese, 1989). We think that our use of osmotically balanced solutions in the $\mathrm{SV}$ isolation was important for having the diameters of the frozen, freeze-substituted isolated SVs match those found in frozen intact nerve endings. Negatively stained, non-fixed isolated SV samples had the largest mean diameter relative to the other samples obtained by different preparation techniques. It should be kept in mind that the negative stain image is a projection of the whole vesicle, while the sections are often only part of a vesicle and not every instance includes the section from the equator. It is also known that when protein-containing lipid vesicles are negatively stained, these vesicles dry down and collapse by approaching the diameter of two disks with the same area, one on top, one below (Szoka and Papahadjopoulos, 1980; Peter, 2010). Changes in vesicle shape from sphere to disk by negative staining would explain the significant increase in negatively stained SV diameter that we observed.

Indeed, such flattening is directly demonstrated in the tomographic reconstructions here (Fig. 3B). With this method, SV membranes appear to be uniformly coated with structures-big knobs and smaller hairs; ostensibly the knobs are the V-ATPase standing out because it is much larger than other SV proteins. Because the stain does not get inside the vesicle, its membrane is not enclosed on both sides, and it is poorly outlined by negative stain in $\mathrm{XZ}$ projections of tomograms, so the membrane appears only as a faint boundary between stain and non-stain. The upper and lower boundaries of vesicles, due to the hairlike structures, are hard to see except where the boundary is decorated by knobs. Here, the knobs clearly delineate the position of the membrane at that point. Thus, knobs on vesicle membranes near the centers of vesicles appear to be on a flat surface suspended across the ends of the vesicles, like the top of a drum, rather than perched on domes, showing that the vesicles are flattened and actually a little thicker at their edges where their membranes fold back on themselves.

It remains to be determined the degree to which detergents used for vesicle isolation in previously published methods and the specificity of metal-protein surface inter- 
actions implicit to the negative staining procedure affect the organization of proteins on the SV surface. We demonstrate here three-dimensional molecular reconstructions based on tomograms from single intact synaptic vesicles isolated without detergents. The tomograms are obtained by negative stain-based electron microscopy, which permits imaging free from the assumptions of symmetry, classification, and averaging. While further research is needed to determine if proteins of specific type may cluster with each other in restricted domains (Bennett et al., 1992), below the structures noted above, negative-staining tomography shows a continuous layer of protein blanketing the surfaces of synaptic vesicles.

In summary, we have optimized the routine collection of relatively large quantities of highly purified synaptic vesicles and performed negative-staining tomography to investigate the distribution of molecules on surfaces of the vesicles. Importantly, the vesicle flattening we detected is likely to result in artifactually enlarged radii of curvature if negative staining is the only measure of diameter in vesicular and tubular systems.

\section{Acknowledgments}

We are grateful to Jane Farrington for examining fractions in the electron microscope, and Dr. Harish Pant for teaching us the optic lobe dissection and homogenization. JAD is supported by the RI-INBRE program award \# P20RR016457-10 from the National Center for Research Resources (NCRR), NIH.

\section{Literature Cited}

Bennett, M. K., N. Calakos, T. Kreiner, and R. H. Scheller. 1992. Synaptic vesicle membrane proteins interact to form a multimeric complex. J. Cell Biol. 116: 761-775.

Bernal, R. A., and D. Stock. 2004. Three-dimensional structure of the intact Thermus thermophilus $\mathrm{H}+$-ATPase/synthase by electron microscopy. Structure 12: 1789-1798.

Carlson, S. S., J. A. Wagner, and R. B. Kelly. 1978. Purification of synaptic vesicles from elasmobranch electric organ and the use of biophysical criteria to demonstrate purity. Biochemistry (Mosc.) 17: $1188-1199$.
Chin, G. J., and S. A. Goldman. 1992. Purification of squid synaptic vesicles and characterization of the vesicle-associated proteins synaptobrevin and Rab3A. Brain Res. 571: 89-96.

Clift-O'Grady, L., A. D. Linstedt, A. W. Lowe, E. Grote, and R. B. Kelly. 1990. Biogenesis of synaptic vesicle-like structures in a pheochromocytoma cell line PC-12. J. Cell Biol. 110: 1693-1703.

Dowdall, M. J., and V. P. Whittaker. 1973. Comparative studies in synaptosome formation: the preparation of synaptosomes from the head ganglion of the squid, Loligo pealii. J. Neurochem. 20: 921-935.

Fox, G. Q. 1988. A morphometric analysis of synaptic vesicle distributions. Brain Res. 475: 103-117.

Huttner, W. B., W. Schiebler, P. Greengard, and P. De Camilli. 1983. Synapsin I (protein I), a nerve terminal-specific phosphoprotein. III. Its association with synaptic vesicles studied in a highly purified synaptic vesicle preparation. J. Cell Biol. 96: 1374-1388.

Kremer, J. R., D. N. Mastronarde, and J. R. McIntosh. 1996. Computer visualization of three-dimensional image data using IMOD. $J$. Struct. Biol. 116: 71-76.

Kretzschmar, S., W. Volknandt, and H. Zimmermann. $1996 . \quad$ Colocalization on the same synaptic vesicles of syntaxin and SNAP-25 with synaptic vesicle proteins: a re-evaluation of functional models required? Neurosci. Res. 26: 141-148.

Peter, B. 2010. Negative-stain spreading of protein-protein and proteinlipid complexes for EM. [Online]. McMahon Lab, Neurobiology Division, MRC Lab of Molecular Biology. Available: http://www. endocytosis.org/techniqs/Negative\%20stain\%20protocol.html [2011, March 3].

Petersen, J. D., X. Chen, L. Vinade, A. Dosemeci, J. E. Lisman, and T. S. Reese. 2003. Distribution of postsynaptic density (PSD)-95 and $\mathrm{Ca}^{2+} /$ calmodulin-dependent protein kinase II at the PSD. J. Neurosci. 23: $11270-11278$.

Pollard, H. B., J. L.Banker, W. A. Bohr, and M. J. Dowdall. 1975. Chlorpromazine: specific inhibition of L-noradrenaline and 5-hydroxytryptamine uptake in synaptosomes from squid brain. Brain Res. 85: 23-31.

Stadler, H., and S. Tsukita. 1984. Synaptic vesicles contain an ATPdependent proton pump and show 'knob-like' protrusions on their surface. EMBO J. 3: 3333-3337.

Szoka, F., Jr., and D. Papahadjopoulos. 1980. Comparative properties and methods of preparation of lipid vesicles (liposomes). Annu. Rev. Biophys. Bioeng. 9: 467-508.

Takamori, S., M. Holt, K. Stenius, E. A. Lemke, M. Gronborg, D. Riedel, H. Urlaub, S. Schenck, B. Brugger, P. Ringler, et al. 2006. Molecular anatomy of a trafficking organelle. Cell 127: 831-846.

Tatsuoka, H., and T. S. Reese. 1989. New structural features of synapses in the anteroventral cochlear nucleus prepared by direct freezing and freeze-substitution. J. Comp. Neurol. 290: 343-357.

Wilkens, S., Z. Zhang, and Y. Zheng. 2005. A structural model of the vacuolar ATPase from transmission electron microscopy. Micron 36: $109-126$ 